\title{
Research on Multi-Source Data Tourism Application Platform in the Minority Area's Scenic Spot
}

\author{
Ying Xiong*, Jing Lin and Wei Wei Qin
}

School of Electronic Information Engineering, South-Central University for Nationalities, Wuhan 430070, China

\begin{abstract}
Through the minority tourism, display platform status and problems of research, we propose a technique based on multi-source data show, this paper based on this technical design of the entire multi-source data application platform overall framework, database, environment and interface able to empty earth integrated perspective view showing the entire ethnic tourism, the multi-source data display technology applications, will the development of the tourism industry throughout the minorities have a greater significance, and has broad market prospects.
\end{abstract}

Keywords: Multi-source data, panoramic roaming, three-dimensional panorama, virtual tourism.

\section{INTRODUCTION}

With the rapid development of the tourism market, tourism already has a historical opportunity of rapid development. Because of the development of information technology, tourism has a revolutionary transformation in scenic area management services, resources protection, marketing, industrial service model and business model in the upstream and downstream industrial chain.

As a unique branch of the tourism, the tourism of Chinese ethnic minorities is in process of extending its influence step by step and strive to exploit minority cultural tourism which multi-faceted shows not only the historical development of the minority nationalities production, residence, catering, clothing, religion, entertainment, sports and marriage but also the modern folk-custom scene. It has made an important contribution to speed up the blossoming in the minority areas and strengthen the unity of all ethnic groups.

The vigorous development of the tourism industry promotes the rapid progress of the construction area. The minority tourism propagandizes its characteristic mainly based in 2D planar media. The audiences only can understand the situation of tourism attractions through the texts, images, videos and other data. Obviously it is difficult to obtain the true feelings in this way which reduces the desire of visiting.

3D panoramic technique photograph was shown in Fig. (1).

The tourism multi-source data network application platform combines with the most popular panoramic view of 3D technology, positioning technology and 3D modeling technology. The way that shows the multi-source data in allround to visitors by using a plurality of windows mode can provides the best pattern to display the minority tourist attractions. What's more, this platform can enormously imbue users with a desire to visit and provide a huge business opportunity for merchants.
At present, China has had the Street View Car represented by SOSO Street View and then turned out tourist attractions in 360 degrees. However, Microsoft's Street Slide connects the photographs into a long strip which are taken on by the Street View Car. Road signs and enterprise data can be shown in the map. Users can "Walking down the street" _ - it was like the sitting on the car for sightseeing. Of course, users also can "stop and go out to admire the beautiful view of the particular scenery". 3D panoramic view street technology has enjoyed its famous at home and abroad, but it is still a virgin area in the minority areas' scenic spot. Displaying interactively of the space, from multiple perspectives (high, low, ground) and multiple sources (panoramic 3D and 2D, 3D models, etc.). Then all the details are assembled to show the whole view of the minority areas' scenic spot. Surely, it will be a very important form of the scenic spot's publicity. Furthermore, it is be of great significance for the development of the minority tourism industry.

This paper develops multi-source displaying platform of the minority areas' scenic spot from panoramic 3D and 3D model, as an exploration of ethnic minority's exhibition in a modal window.

\section{SYSTEM DESIGN}

\subsection{System Construction Goal}

Overall goal is the use of geographic information system data (includes panoramic images data, topographic data, remote sensing data, geographical names data, etc), the landscape data, tourism and related data based on network of computer and spatial database which achieve the application platform of multi-source data network travel. The platform has the features of centralized management and unified update maintenance, providing the travel integrated services platform with intuitive, dynamic, interactive, content-rich information. Ultimately the platform achieve the goal that can show the scenic area such as displaying 2D or 3D style, querying travel roaming information, simulating the tourist routes and viewing the multimedia information [1-3]. 


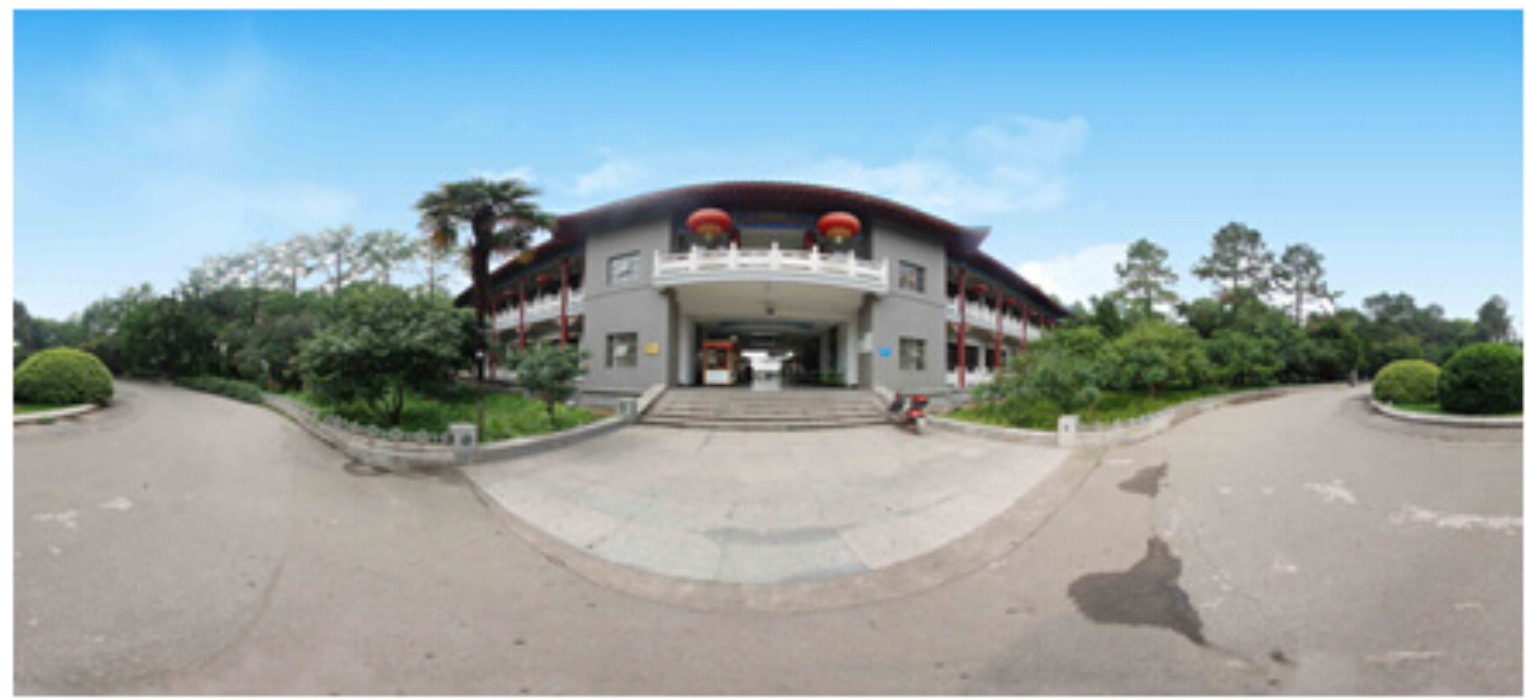

Fig. (1). 3D panoramic technique photograph.
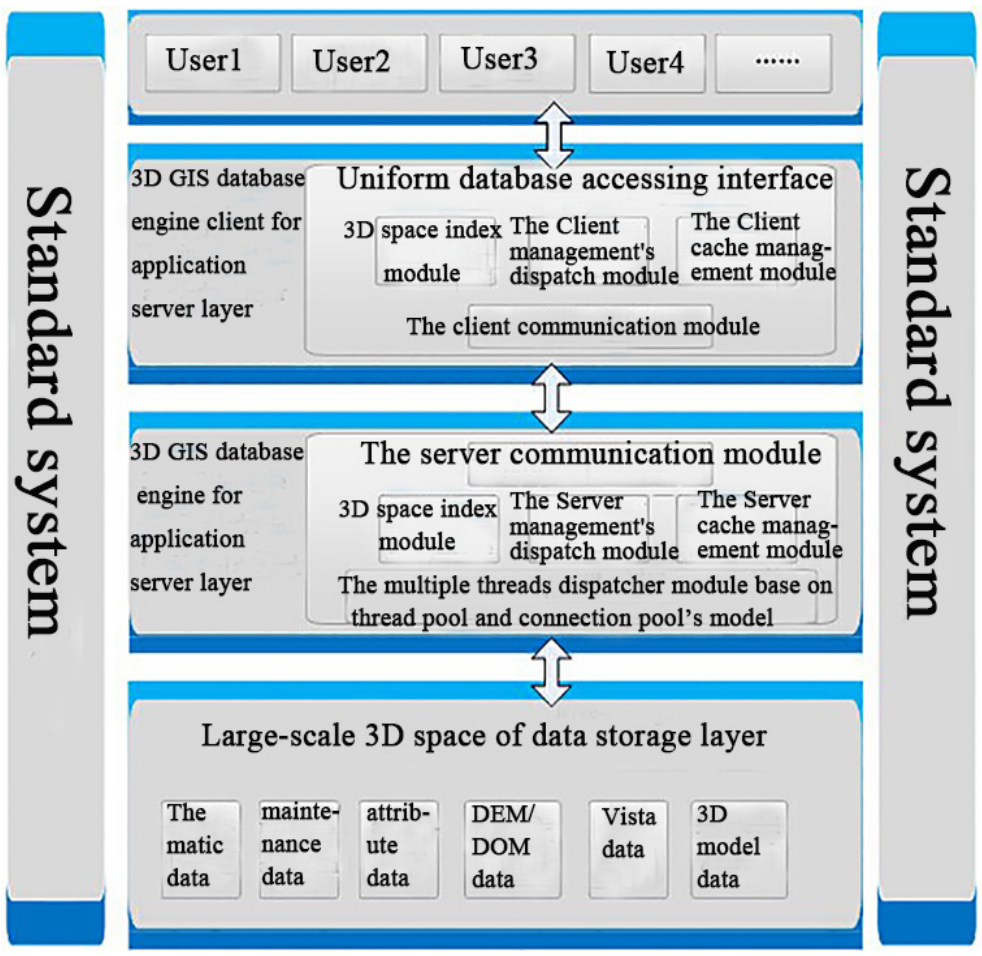

Fig. (2). System architecture.

\subsection{Framework and Main Functions of the System}

\subsubsection{System Architecture}

The system of platform is shown in Fig. (2). The platform adopts three-tier system of $\mathrm{B} / \mathrm{s}$ mode. It is divided into three layer on the basis of technical structure, including the user layer, middle layer (business logic) and data services layer. The 3D module uses the second-development interface and control to develop that is provided by Terra Explorer Pro of Skyline (the 3D geographic information platform). The 2D module uses ArcIMS and ArcEngine that is provided by ArcGIS to develop, also need to use ArcIMS and WFS (Web Map Service). Panoramic map module is based on the Open Source Components Gmap.net to actualize functions.

\subsubsection{The Main Functions of the System}

The functional classification of multi-source data tourism network application platform is shown in Fig. (3). It will conducting the description according to the business functions, including the following features.

\section{(1) 3D model browse}

The national scenic area show based on 3D model. Compared with the traditional image, viewing the scenic area in $3 \mathrm{D}$ engine can get more abundant tourist information. Visitors can be exposed to (indulge) dynamic real image of various attractions. By clicking the mouse in person to manipulate, roaming the minority areas' scenic spot, by the way 


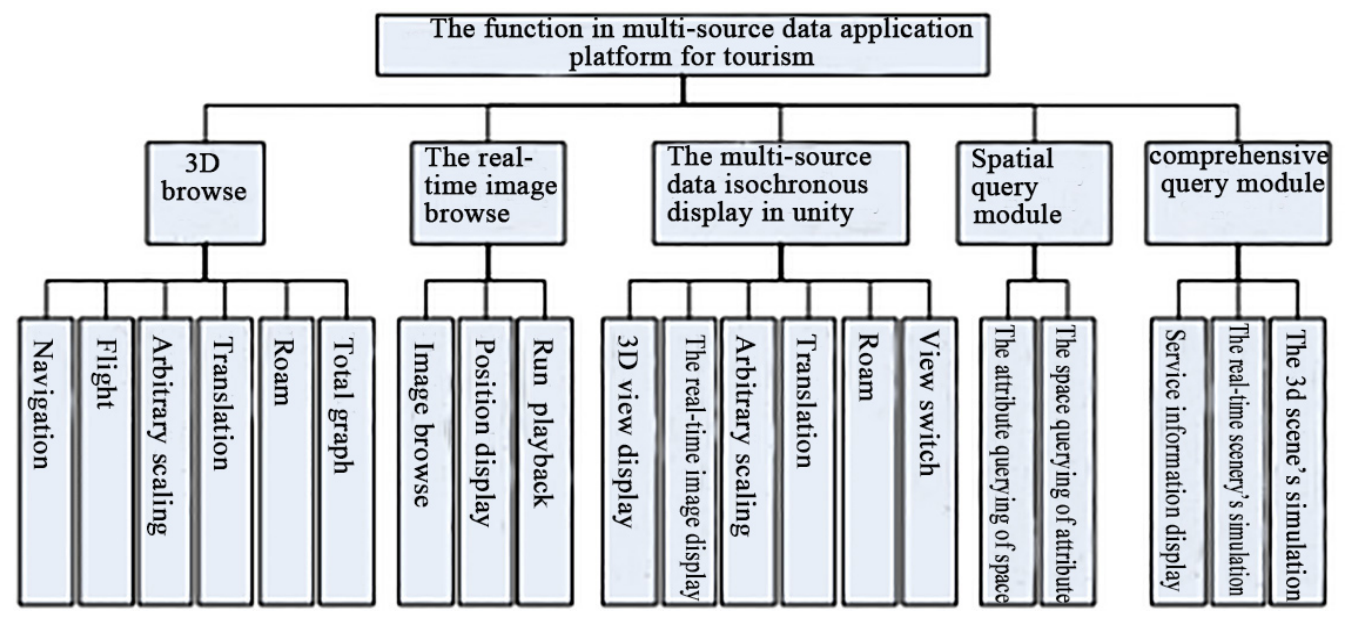

Fig. (3). The multi-source data application platform function of tourism.

they can know attractions or the future direction of urban planning and development of the city.

3D model display gives the attractions to living life. Viewing attractions in the $3 \mathrm{D}$ model is more abundant than the traditional way or 2D image display, including navigation, flight, zoom, Pan, roaming, interactive map showing. Users can observe the orientation of the surface features' forms from different perspectives [4].

\section{(2) Panoramic image browse}

Panoramic images are snapshots of the real world. It truly records the information of geographical environment landscape and geographic spatial information in photographing. Can see is the same with the real world. Tourist attractions use panoramic image data to greatly enhance the tour experience. This not only can be used for evaluation of tourist information, also can review the runs, enhance the tourist area's awareness, increases the amount of visitors [5]. play

(3) The integration of multi-source data synchronous dis-

The view of the system is divided into $3 \mathrm{D}$ views, panoramic views and $2 \mathrm{D}$ views. Achieving any combination can be shown or hided. In any windows it provides the basic operation, such as enlarge, and reduce, roaming and map. Selecting an object in the 2D map and switching to the 3D views or panoramic images, the perspective of objects dives to the $3 \mathrm{D}$ environment form the air. Meanwhile you can still display its attribute information in 3D state, allowing users to have more profound and at the visual impression.

\section{(4) Spatial query module}

Spatial querying is the most basic and most commonly function in GIS [6]. Spatial object by using the mouse to click on the map or using the keyboard enter a keyword query property information for points of interest, from space to a property, from property to space the bi-directional queries [7].

(5) The query and display module of tourism information

According to "transportation, accommodation, travel, food, shopping and entertainment" is organized to provide scenic spots and around all kinds of services, opening hours, contact details, information on rates and other travel services. General services is dominated by text and picture shows, key regions being provided in the form of videos.

Also offers the tourist routes of panoramic or $3 \mathrm{D}$ scene simulation, the generated plurality of panoramic and 3D scene route in the system. Tourists select specified video [8], and it demonstrated in the multi-source data view shows.

\subsubsection{System Database Design}

The platform contains two types of data: one is the fundamental geographic information data, and another is the integrated travel information data. The basic geographic information data include a variety of high-resolution remote sensing image (DOM), digital elevation model (DEM), panoramic imagery, linear vector data, geographical names data, building models data, and so on. The comprehensive tourism information data is divided into spatial data and attribute data, comprising: vector data, text data, graphics, video, sound etc. According to the requirements of data integration, through spatial data and attribute data for effective integration, actualize graphic and attribute efficient mutual checking.

The entry of basic information data needs to maintain a uniform spatial reference. At the same time due to the Internet access, access speed is a factor which are suppose to be considered. Therefore, all data are needed for Pyramid cutting. After cutting the tile folder named is not unique. In order to the facilitate calculation, intends to use the value of the zoom level as the name of each set of Tile Map Database. Experiments shows that if the map tiles of each level is stored simply in the same database tables, in addition to meeting management and storage constrained problem, the client access speed on the map tiles being going to slow down $[9,10]$. So it is necessary to establish an index mechanism to enhance the search speed of the Pyramid shaped mass map tiles database. Because each map tiles file's names are it's the identification of geographical location information. In the cutting process of map tiles, assuming that naming after the way of $\mathrm{x} \_\mathrm{y}$, current_longitude, current_latitude represent longitude and latitude, current_level represent the level of zoom. With javascript to describe, then the indexing algorithm can be summarized as follows: 


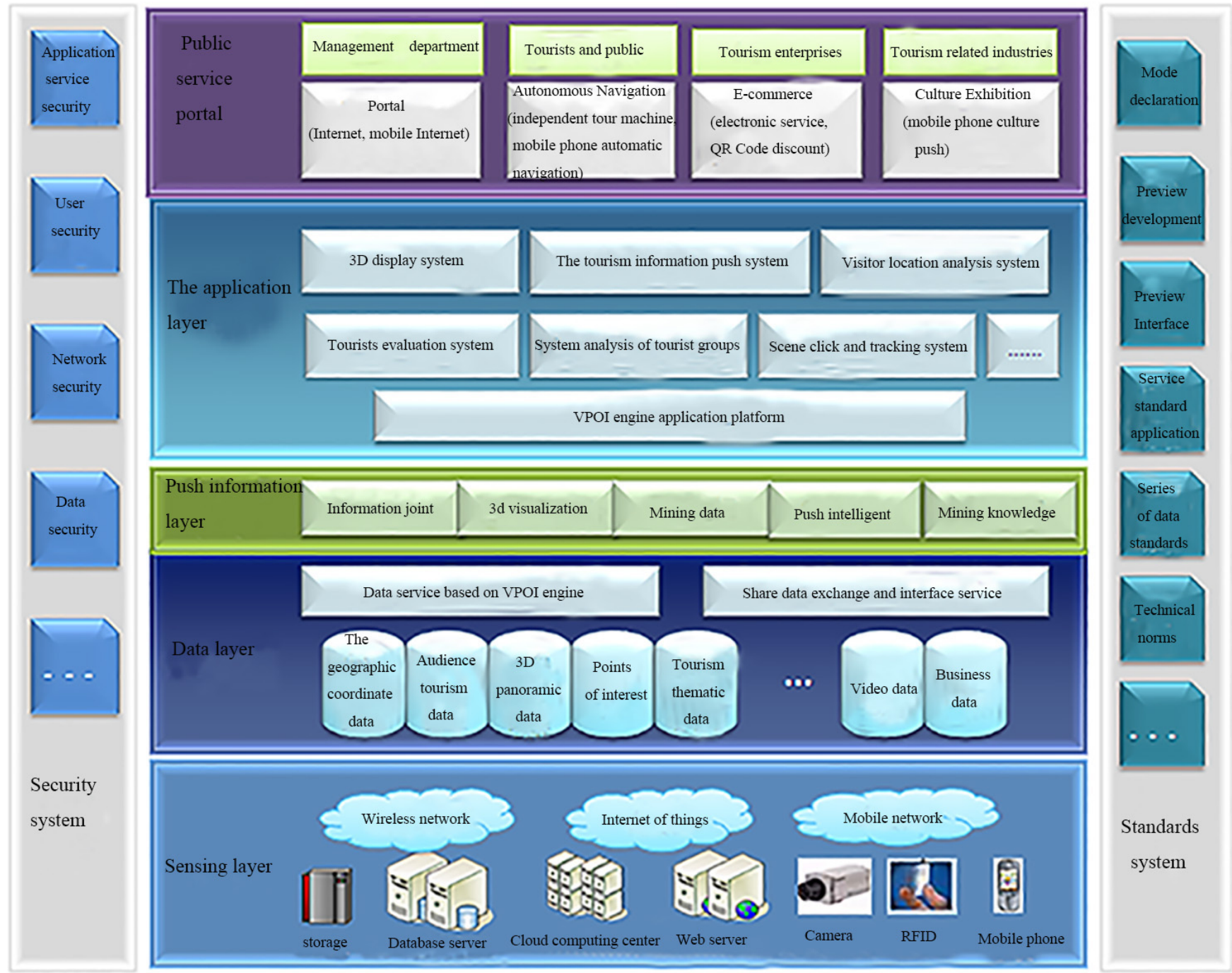

Fig. (4). The overall design of the schema map.

$$
\begin{aligned}
& x=\frac{\text { current_longitude }}{\text { longitude_steps[current_level] }} \\
& y=\frac{\text { current_latitude }}{\text { latitude_steps[current_level] }}
\end{aligned}
$$

Construction of tourism information data contain spatial data and attribute data's construction. The construction of the spatial data is converting the point (place-name), line (tour route), surface (planning project) and other data in projection to SHP file, and imported into SDE spatial database. The construction of the attribute data is descriptive information about the various spatial data imported from the backend data management platform, then using the homegrown data editing gadgets to associate them. Spatial data is stored in Oracle through the ArcSDE spatial database engine. And attribute data is stored directly in Oracle. They are connected by the exclusive OID code.

\subsubsection{System Platform}

Focusing on the ethnic tourism information recommendation system based on VPOI is building data integration and promotion platform for tourist information.
The informational promotion platform of ethnic tourism which based on VPOI engine will use visual technology to realize the intelligent tourism information push by threedimensional panorama. The back-end data service engine have realized aggregate information module, mining knowledge module, intelligent push information module and so on. The front-end data service engine have achieve visual display and interactive modules, including browse, roam, navigation, tag, comments, virtual interactive etc, which based 3D panorama.

\subsubsection{Data Mining}

VPOI engine is the core technology of data mining for data integration and mining. That is from the massive visual depth of analysis, data mining in the POI information, provide the basis for intelligent push.

\subsubsection{Knowledge Engine}

Knowledge of engine is built on the basis of VPOI database, through continuous interaction with the user, combined by the user's own upload the knowledge base and the engines, establishing a knowledge engine and providing the optimal solutions to the user. 


\subsection{Visualization}

Using 3D panorama technology will be interested in the visual scene collection, database. In this topic we use the hexahedral model for modeling. The using of panoramic lens (fish - eye lens) or ordinary continuous camera shot a 180 degree horizontal space 4 photos 2 photos and 180 degree angle of vertical space, will be given on hexahedron model 6 photo, forming $3 \mathrm{D}$ visualization scenes.

\subsubsection{Information Aggregation}

Information aggregation automatic collection of information combine with artificial way to improve the rate of accurate interpretation.

\subsubsection{Intelligent Push (PDA, Sensors, and so on)}

Based on parameters such as user habits and location intelligent push support different terminals.

\subsection{Environmental Design}

The platform use Asp.net as a development environment and needs 4 servers in total in the process of construction which are respectively WFS servers, TerraGate servers, database servers, and WEB servers. The database server installs software that named Oracle, and it is used to manage the various scenic spatial data and attribute data. WFS server installs software including ARCIMS, ARCGIS, Apach etc. The software are used to deliver map data, mainly supplying 3D topographic data for dissemination service. The TerraGate server installs IIS, asp.net, skyline which can organize and publish the 3D data. The WEB server installs IIS, Asp.net and it provides internet service for public. The platform server deploy as shown in the Fig. (4).

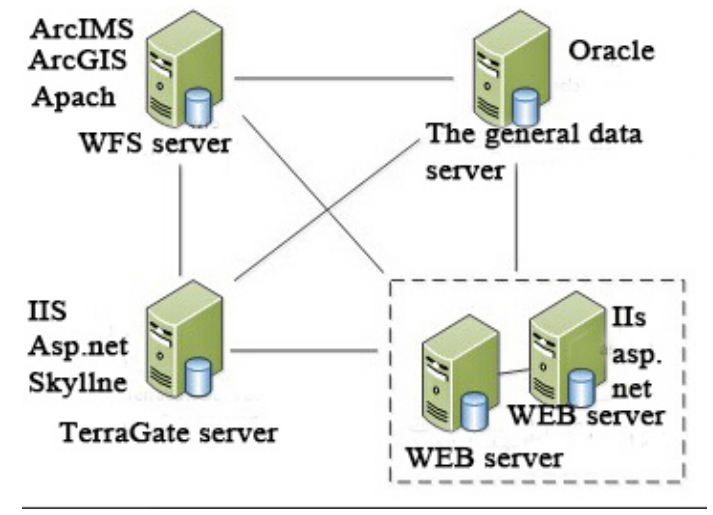

Fig. (5). The deployment of platform server.

\subsubsection{WFS Service Development}

The WMS/WFS service provides description of the exploitation which support WMS (Web Map Service)/WFS (Web Feature Service) service interface specification formulated by OGC (Open GIS Consortium), and defines IMS service interface specification to support the WMS/WFS service specification. The service actualize interoperability between heterogeneous and WebGIS systems, and real-time mapping and vector data manipulation. Configuration files as follows:

$<$ Extension $>$
$<$ TypeName $>$ WFSServer $<$ TypeName $>$

$<$ Enabled $>$ true $<$ /Enabled $>$

$<$ Properties $>$

$<$ AxisOrderWFS11 $>$ longlat $<$ /AxisOrderWFS11 $>$

$<$ CustomGetCapabilities $>$ false $</$ CustomGetCapabilities $>$

$<$ EnableTransactions $>$ false $</$ EnableTransactions $>$

$<$ Name $>$ wfs_WFSTest_ras $</$ Name $>$

$<$ OnlineResource $>$ http://bobmk/arcgis/services/wfs/ WFSTest_ras/MapServer/WFSServer $</$ OnlineResource $>$

$<$ AppSche-

maURI>http://bobmk/arcgis/services/wfs/WFSTest_ras/Map Server/WFSServer $</$ AppSchemaURI $>$

$<$ AppSchemaPrefix $>$ wfs_WFSTest_ras $<$ _ AppSchemaPrefix $>$

$$
\begin{aligned}
& <\text { /Properties }> \\
& <\text { Info }> \\
& <\text { WebEnabled }>\text { true }<\text { /WebEnabled }> \\
& </ \text { Info }> \\
& </ \text { Extension }>
\end{aligned}
$$

\subsubsection{Load Balancing}

Load balancing is composed of multiple services in a symmetrical way forming a collection of servers. Each server has the equivalent status, providing service independently without other servers' auxiliary. The external request will be evenly distributed to the symmetrical structure on the one server, and the server that receives the request is able to independently response to client's requests. Local balancing can evenly distributed client requests to the server array in order to access quickly for vital data. In this way and solve concurrent service issues with numerous access. This clustering technology gets performance that close to mainframe with minimal investment.

The platform uses two servers as WEB server, publishing terrain data, while achieving load balancing, fast access for multi-user concurrent. As shown in the Fig. (5).

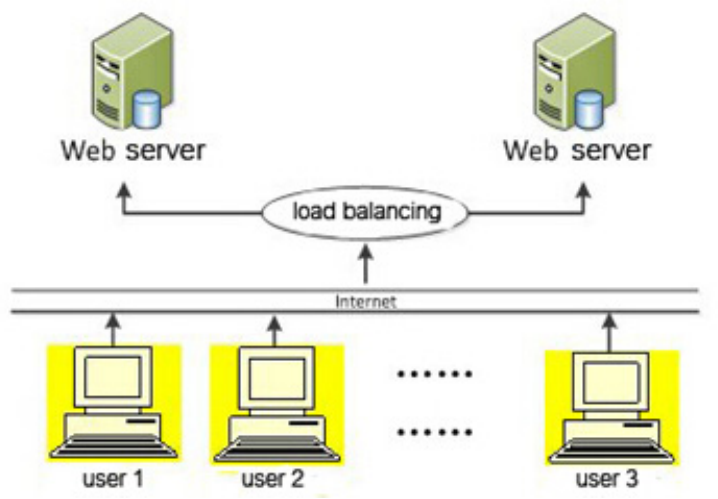

Fig. (6). Diagrammatic sketch for load balancing.

Diagrammatic sketch for load balancing was shown in Fig. (6). This platform uses Nginx+iis to realize the load balance, the nginx.conf settings are as follows: 


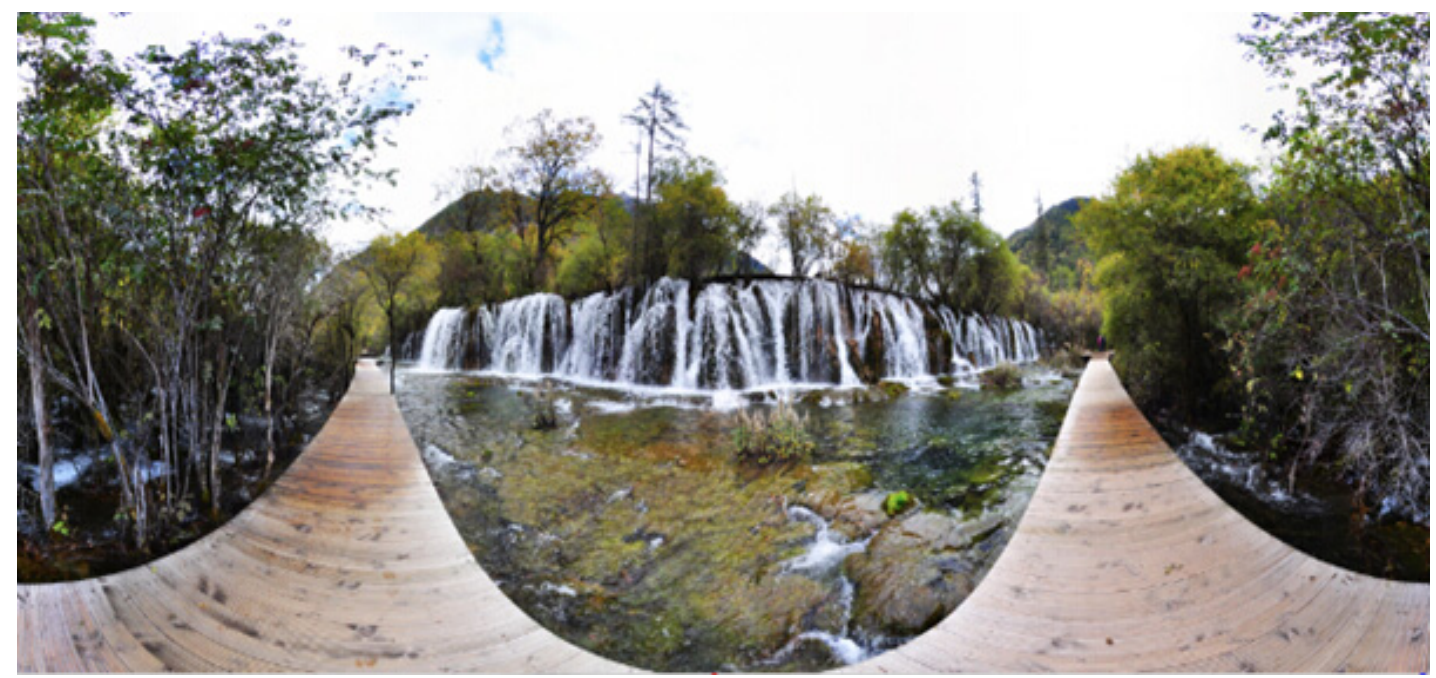

Fig. (7). The system operation interface diagram.

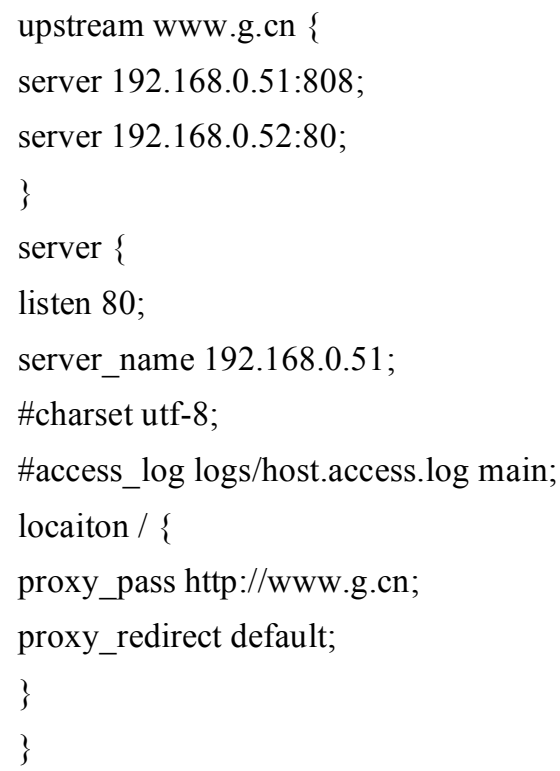

\subsection{Interface Example}

The minority areas' scenic spot module of multi-source data tourism platform include scenic points, routes, hotels, shopping, entertainment, tour guides etc [9]. The multisource data window displays comprise $3 \mathrm{D}$ model window display, panoramic images window display, 2D pictures window display. Those window displays use GPS to matching location, showing a scenic point's 3D model, panoramic images and 2D location. From multiple angles, multiple dimensions to show the scenic outlook, it is extremely good for users to experience $[10,11]$. The system operation interface diagram was shown in Fig. (7).

\section{CONCLUSION AND PROSPECTION}

The multi-source data tourism application platform for minority areas' scenic spot including information system of traditional tourism industry is mainly based on $\mathrm{B} / \mathrm{S}$ mode, business and information exhibited to the user by the PC browser. The next generation tourism platform will be more lie on the mobile and portable (mobile phone, tablet PC, portable equipment, etc.). Using the up-to-date mobile Internet's platform terminal and mobile intelligent system (android/ios), combine with the current EPC system network, cloud computing, $3 \mathrm{G}$ mobile communication, multimedia technology, digital tourism technology to construct mobile intelligent tourism information platform and application system.

Intelligent ethnic tourism information platform can realize the function of mobile terminal, such as choosing the closest route or the suggestions of the closest route, the mobile application that based on streaming media services, attractions HD video playback (using the free wifi), attractions surveillance camera video sharing, self-guided, parking guidance, etc. The minority tourism management will be ascended to a height of digital and intelligent.

\section{CONFLICT OF INTEREST}

The authors confirm that this article content has no conflict of interest.

\section{ACKNOWLEDGEMENTS}

(1) Foundation of the Fundamental Research Funds for the Central Universities, South-Central University for Nationalities (CZY14002).

(2) Foundation of Agricultural Technology Research and Development Laboratory Accommodation of Chinese Academy of Sciences and State Ethnic Affairs Commission.

(3) Foundation of Agricultural Technology Research and Development Laboratory Accommodation of Chinese Academy of Sciences and State Ethnic Affairs Commission.

(4) Foundation of National Natural Science Foundation of Chinese State Ethnic Affairs Commission (12ZNZ023).

(5) Foundation of the National Training Programs of Innovation and Entrepreneurship for Undergraduates (GXX14217).

\section{REFERENCES}

[1] S.E. Chen, "QuickTime VR-an image-based approach to virtual environment navigation," In: IEEE Transactions on Computer Graphics, 1995, pp. 29-39. 
[2] X. Zhang, Z. Wu, and L. Song, "The Design and Implement of Virtual Panorama System," Chinese Conference on System Simulation Technology Application, vol. 12, pp. 64-67, 2010.

[3] S. Richard, "Image Alignment and Stitching: A Tutorial," Technical Report, MSR-TR-2004-92, vol. 10, pp. 1-69, 2006.

[4] J. Matas, O. Chum, and M. Urban, "Robust wide baseline stereo from maximally stable extremal regions," Image and Vision Computing, vol. 22, no. 10, pp. 761-767, 2004.

[5] M. Fischler, and R. Bolles, "Random Sample Consensus: A Paradigm for Model Fitting with Applications to Image Analysis and Automated Cartography," Communications of the ACM, vol. 24, pp. 381-385, 1981.

[6] J. Davis, "Mosaics of Scenes with Moving Objects," In: IEEE Conference on Computer Vision and Pattern Recognition (CVPR), 1998, pp. 354-360.
[7] S. Pastoor, and M. Wopking, "3-D displays: a review of current technologies. Displays," vol. 17, no. 2, pp. 100-110, 1997.

[8] J.Y. Son, B. Javidi, and K.D. Kwack,"Methods for displaying three dimensional images," In: Proceedings of the IEEE, vol. 94, no. 3, pp. 502-503, 2006

[9] J.H. Park, S.Y. Jung, H.J. Choi, and B. Lee, "Viewing angle enhanced integral imaging by elemental image resizing and elemental lens switching," Applied Optics, vol. 41, no. 32, pp. 6875-6883, 2002.

[10] Y. H. Kim, J. H. Park, H.J. Choi, S. Jung, S.W. Min, and B. Lee, "Viewing angle enhanced integral imaging system using a curved lens array," Optics Express, vol. 12, no. 3, pp. 421-429, 2004.

[11] H. Noto, and A. Hashimoto, "Relief-like depth map generation algorithm," The Institute of Image Information and Television Engineers, vol. 59, no. 10, pp. 41-49, 2005.

Received: September 22, 2014

Revised: November 30, 2014

Accepted: December 02, 2014

(C) Xiong et al.; Licensee Bentham Open.

This is an open access article licensed under the terms of the Creative Commons Attribution Non-Commercial License (http://creativecommons.org/licenses/by-nc/3.0/) which permits unrestricted, non-commercial use, distribution and reproduction in any medium, provided the work is properly cited. 